\title{
Correction to "The Relationship of Hepatitis Antibodies and Elevated Liver Enzymes with Impaired Fasting Glucose and Undiagnosed Diabetes"
}

In the abovementioned article (Mainous AG, Diaz VA, King DE, Everett CJ, Player MS.J Am Board Fam Med 2008;21(6):497-503), the last line in the results section of the abstract should read: "Hepatitis antibodies, reporting a current liver problem, or AST were not associated with having undiagnosed diabetes in adjusted analyses." We apologize for the error, and we regret any confusion or inconvenience it may have caused. (doi: 10.3122/jabfm.2009.02.080246.) 